\title{
幸福感的静息态功能磁共振成像低频振幅
}

\author{
罗扬眉 ${ }^{(1) 2}$ ，李宝林 ${ }^{(1)}$ ，刘杰 ${ }^{(1)}$ ，毕重增 ${ }^{(1)}$ ，黄希庭 ${ }^{(1 *}$ \\ (1) 西南大学心理学部, 教育部认知与人格重点实验室, 重庆 400715; \\ (2) 陕西师范大学心理学院, 西安 710062 \\ * 联系人, E-mail: xthuang@swu.edu.cn
}

2014-07-07 收稿, 2014-08-15 接受, 2014-12-16 网络版发表

西南大学心理学部 2012 研究团队建设项目和西南大学重庆市人文社会科学重点研究基地项目(TR201201-1)(14SKB008)资助

\begin{abstract}
摘要 人人都想得到幸福, 但并不是每个人都有相同的获得幸福的能力. 对于幸福个体差异这 一重要而又有意义的问题, 以往很少有研究来探索该问题的神经起源. 本研究采用静息态功能 磁共振成像中的低频振幅(amplitude of low frequency fluctuations, ALFF)技术, 以 159 名健康大 学生被试为对象, 研究了幸福感与大脑自发神经活动之间的关系. 结果发现, 额叶、顶叶以及 部分皮层下区域的 ALFF 值与幸福感有紧密关系。具体地说, 左、右侧眭额皮层和左侧背外侧 前额皮层, 以及右侧尾状核的 ALFF 值随着幸福感的增加而升高, 并且这些区域的 ALFF 值都 与情绪有显著相关. 相反, 右侧中扣带皮层, 左侧顶上沟以及双侧楔前叶的 ALFF 随着幸福感 的增加而降低. 这些区域的 ALFF 值不仅与情绪有关, 而且还与沉思的倾向有关系. 这些大脑 区域的 ALFF 值与幸福感的关系可能表明了高低幸福感个体在自我反思、奖赏加工、情绪调节 等能力上存在显著差异.
\end{abstract}

关键词

幸福感

静息态

低频振幅

快乐

情绪调节

沉思
幸福是人类所追求的共同目标. 几乎人人都想 要得到幸福 ${ }^{[1]}$, 但是并不是每一个人都具有同样的获 得幸福的能力. 在日常生活中, 我们不难发现, 有的 人即使身处逆境却依然感到很幸福, 而有的人尽管 处境优越却还是感到不幸福. 对于这样一个令人着 迷而又非常重要的幸福个体差异问题, 心理学家们 从认知、人格、社会等各个角度进行了诸多研究 ${ }^{[2 \sim 4]}$. 例如, 幸福的建构理论认为, 高幸福个体和低幸福个 体在多种认知和动机过程上存在系统性差异, 包括 自我反思、自我评价、社会比较和人际知觉等维度 ${ }^{[4]}$. 近年来, 研究者们也开始尝试从神经科学的角度来 研究幸福感存在个体差异的神经根源 ${ }^{[5]}$. 例如, 最近, 两项基于任务的功能磁共振成像(functional magnetic resonance imaging, fMRI)研究关注了高低幸福感个 体情绪加工过程的神经机制, 结果发现高幸福个体 相对于低幸福感个体对积极情绪刺激在杏仁核上有
更大的神经活动 ${ }^{[6]}$, 并且在纹状体和背外侧前额皮层 的神经反应能够维持较长时间 ${ }^{[7]}$.

然而, 正如前两项研究只是关注高低幸福感个 体的单一维度(情绪加工), 采用传统的基于任务的 fMRI 技术来研究幸福感存在一个很大的挑战, 即很 难设计出能够引发全面的幸福体验的任务. 但是, Biswal 等人 ${ }^{[8]}$ 的静息态功能磁共振成像方法有效地 解决了这一难题. 该方法不受任务的制约, 测量的是 人脑在休息状态下血氧水平依赖低频波动信号, 反 映了人脑自发神经活动. 它具有较高的稳定性, 能较 好地反映个体差异 ${ }^{[9,10]}$, 能够有效用来研究人脑内在 功能架构, 已经被广泛用于研究各类精神类疾病, 如 抑有症、精神分裂症等 ${ }^{[11 ~ 13]}$. 在众多静息态功能磁共 振指标中，低频振幅 (amplitude of low frequency fluctuations, ALFF)采用一个频段 $(0.01 \sim 0.08 \mathrm{~Hz})$ 内所 有频率点上幅值的平均值来表征一个体素自发神经

引用格式: 罗扬眉, 李宝林, 刘杰, 等. 幸福感的静息态功能磁共振成像低频振幅. 科学通报, 2015, 60: 170-178 Luo Y M, Li B L, Liu J, et al. Amplitude of low-frequency fluctuations in happiness: A resting-state fMRI study (in Chinese). Chin Sci Bull, 2015, 60: 170-178, doi: 10.1360/N972014-00700 
元活动的强弱, 从能量的角度反映了各个体素在静 息状态下自发活动水平的高低 ${ }^{[14]}$. 它不需要依赖任 何外在时间信息和模型, 是一种可靠的数据驱动的 方法. 以健康被试为研究对象的研究发现, ALFF 不 仅能够反映个体不同的状态 ${ }^{[15]}$, 而且还能够反映出 个体长期的稳定的特质 ${ }^{[16,17]}$. 并且, 它已经在临床上 得到广泛应用, 如注意缺陷多动障碍 ${ }^{[14]}$ 、阿尔兹海默 病 $^{[18]}$ 、轻度认知损害 ${ }^{[19]}$ 、精神分裂症 ${ }^{[20,21]}$ 以及抑郁 症 ${ }^{[22]}$ 等. 因此, 本研究拟采用静息态功能磁共振中 的 ALFF 技术来研究幸福感与大脑的关系.

幸福感是指评价者根据自定的标准对其生活质 量的整体性评估. 它是一个复杂的建构, 包含了很多 子心理成分. 至于幸福感到底由哪些成分构成, 研究 者们并没有一致的意见. 但是, 从以往研究中得知, 高幸福感和低幸福感个体之间在快乐感、情绪调节和 自我反思(self-reflection)上存在系统性差异 ${ }^{[4,5,23]}$. 个 体获得快乐感的能力, 组成了幸福感的核心, 也是获 得幸福感的必要条件. 相反, 如果个体得不到快乐, 就会引发诸多情感障碍, 如抑郁、成瘾和精神分裂 ${ }^{[24]}$. 参与快乐感加工的核心大脑结构之一是基底核(basal ganglia，包括纹状体、丘脑下核和黑质 $)^{[25]}$. 我们的一 项研究采用了静息态 fMRI 技术对高幸福感个体和低 幸福感个体的局部一致性进行了比较. 结果发现, 高 低幸福感个体在纹状体的局部一致性上存在差异 ${ }^{[26]}$. 基于这些证据，我们假定，参与快乐加工的脑区，如 基底核，其 ALFF 值可能会与幸福感有着密切的 关系.

当然, 快乐感只是幸福感的一部分, 不能完整描 述幸福感这一复杂的建构, 幸福感还与良好的情绪 调节能力有关 ${ }^{[23,27,28]}$. 情绪调节指的是个体对情绪的 发生、体验进行调控的能力. 良好的情绪调节能力能 够有利于个体保持愉快的心境、改善不利的心境. 因 此, 它对个体的幸福感有着重要的意义. 例如, Pe 等 人 ${ }^{[23]}$ 发现高幸福感个体能够较好地在工作记忆中保 持和更新积极信息. 情绪调节主要是由前额皮层等 大脑区域参与. 由此, 我们推测, 参与情绪调节的前 额皮层的 ALFF 值可能也会与幸福感有关系.

除了快乐感和情绪调节之外, 自我反思也与幸 福感有密切关系. 过度的负面自我反思, 即过度地关 注自我的消极情绪、问题和其他消极体验，对幸福感 有负面的影响. 特别的是, 沉思(rumination), 即反复 地关注自己的抑郁症状以及并思考这些症状的原因
和可能后果, 是造成不幸福(抑郁)的主要因素之一 ${ }^{[29]}$. 例如, Killingsworth 和 Gilbert ${ }^{[30]}$ 利用手机应用程序收 集了 2250 个被试的实时自我报告, 结果发现那些越 容易离开当下任务而去自我沉思的个体越不快乐. Lyubomirsky 等人 ${ }^{[31]}$ 发现, 低幸福感个体比高幸福感 个体对任务成绩的负性反馈更加敏感，在随后的任 务中表现出了过度的自我沉思，而这种沉思影响了 该任务的成绩. 神经科学的研究已经发现，在静息状 态下与自我相关的加工主要是由默认网络 (default mode network, DMN)参与 ${ }^{[32,33]}$. 默认网络主要包括 内侧前额皮层(medial prefrontal cortex, MPFC)、后扣 带皮层 (posterior cingulate cortex, PCC) 和楔前叶 (precuneus). 它在大脑处于休息状态下被激活，在从 事外部任务的时候被抑制 ${ }^{[34,35]}$. 在理论上, Kringelbach 和 Berridge ${ }^{[5]}$ 认为默认网络由于与自我反思有关, 会与幸福感有着密切关系; 在实证上, 我们的研究也 发现与默认网络有关的脑区，如内侧前额皮层和后 扣带皮层的局部一致性在高低幸福感个体之间存在 差异 ${ }^{[26]}$. 总的来说, 我们假设, 默认网络的重要节 点的 ALFF 值也会与幸福感存在联系, 并且这种联系 是与个体的沉思倾向有关的.

基于这些假设, 本研究采用静息态 fMRI 中的 ALFF 作为指标，扫描了 159 名健康大学生在静息状 态下的大脑自发神经活动，并采用问卷测量了被试 的幸福感, 对幸福感与大脑自发神经活动的关系进 行了研究.

\section{1 方法}

(i ) 被试. 本研究数据来源于时间与人格脑成 像数据库. 其中, 已经有 51 个脑成像数据已经发 表 ${ }^{[28]}$. 一共有 168 名西南大学在校本科生或研究生 参与扫描. 所有被试身心健康，均为右利手，视力或 矫正视力正常. 所有被试实验前均签署知情同意书, 实验结束后给予一定报酬. 所有实验均得到西南大 学心理学部伦理委员会批准.

考虑到头动对静息态数据质量的影响，有 7 名被 试由于头动过大其脑成像数据被删除(删除标准见下 文). 另外 2 名被试由于没有参与后期的问卷调查, 数据也被删除. 最终剩下 159 名被试的有效数据. 其 中，男 66 名，女93名. 年龄范围在 18 25 岁之间，平 均年龄为 $20.90 \pm 1.64(M \pm S D)$.

(ii) 中国人幸福感量表(Chinese happiness in- 
ventory, CHI). 采用 $\mathrm{Lu}$ 等人 ${ }^{[36]}$ 编制的中国人幸福感 量表完整版测量了所有被试的幸福程度. 该量表一 共 48 个题项, 分为乐观、社会承诺、正向情感、掌 控感、身体健康、自我满足、心理警觉、和谐的亲友 关系、他人赞赏、物质满足、工作成就、活得比别人 好、乐天知命等 13 个纬度. 在本研究中, 该量表的 Cronbacha's $a$ 系数为 0.943 .

(iii) 积极和消极情感量表(positive and negative affect schedule, PANAS). 该量表是由 Watson 等 人 ${ }^{[37]}$ 编制的, 用于测量被试的情绪状态. 由于扫描 程序的安排问题, 只有 118 名被试参与了情绪状态的 测量. 该量表包含 2 个分量表, 即积极情感分量表和 消极情感分量表, 分别有 10 个条目. 每个条目采用 1 5 评分. 该量表的中文版被证实具有良好的信效 度 ${ }^{[38]}$. 在本次实验中, 积极和消极情感量表的 Cronbacha's $a$ 系数为 0.770 .

(iv) 沉思反应量表(rumination response scales, RRS). 采用沉思反应量表 ${ }^{[39]}$ 测量了被试的沉思倾 向. 其中, 72 名被试测量了该量表. 该量表包含 3 个 维度: 抑郁(depression)、强迫性冥思(brooding)和反思 (reflection) 测量. Treynor 等人 ${ }^{[39]}$ 认为, 沉思可以分成 强迫性冥思和反思 2 个因素, 因此在本研究中只用了 这 2 个分量表. 强迫性冥思是指个体消极地和理想标 准做比较的倾向; 反思是指个体试图解释自己为什 么心境低落原因的倾向. 该量表已经在中国有适用 性研究, 其中文版有 21 个条目, 1 4 级评分, 符合心 理测量学标准 ${ }^{[40]}$. 在本研究中, 强迫性冥思和反思 的 Cronbacha's $a$ 系数分别为 0.65 和 0.78 .

(V) fMRI 扫描. 数据采集使用 $3.0 \mathrm{~T}$ 磁共振成 像系统(Siemens Magnetom Trio Tim). 在标准的头线 圈内完成扫描, 扫描时用配套的泡沫固定头部位置, 以减少被试的头动. 全脑功能像由 $\mathrm{T} 2^{*}$ 加权单次激发 梯度回波的 EPI 序列获得, 功能像相关参数如下: TR $=2000 \mathrm{~ms}, \mathrm{TE}=30 \mathrm{~ms}$, 反转角 (flip angle) 为 $90^{\circ}$, 扫 描视野 $(\mathrm{FOV})=220 \mathrm{~mm} \times 220 \mathrm{~mm}$, acquisition matrix $=64 \times 64$, 轴位 32 层, 层厚 $=3.0 \mathrm{~mm}$, voxel size $=3$ $\mathrm{mm} \times 3 \mathrm{~mm} \times 3 \mathrm{~mm}$. 在扫描过程中, 要求被试闭上 眼睛, 不要睡着, 不要想特别的事情. 对于每一个被 试, 收集 $8 \mathrm{~min}$ 的静息态数据, 共获得 240 个时间点. 结构像扫描参数: $\mathrm{TR}=1900 \mathrm{~ms}, \mathrm{TE}=2.52 \mathrm{~ms}$, 轴位 176 层, 层厚 $=1.0 \mathrm{~mm}$, 层间距 $=0.5 \mathrm{~mm}$, flip angle 为 $90^{\circ}, \mathrm{FOV}=256 \mathrm{~mm} \times 256 \mathrm{~mm}$. (vi) 数据预处理. 在 Matlab 平台上使用基于 SPM8 (http: //www.fil.ion.ucl.ac.uk/spm)的 DPARSFA (data processing assistant and resting-state FMRI advanced, version 2.2, http://www.restfmri.net/forum/ DPARSF $)^{[41]}$ 软件来对数据进行预处理. 预处理步骤 如下: (1) 为了获得稳定的磁共振图像和被试适应磁 共振环境, 将功能像数据前 10 个时间点剔除. (2) 时 间层配准, 以校正 slice 获取的时间差. (3) 头动矫正. (4) 将 $\mathrm{T} 1$ 像分割成灰质、白质和脑脊液. (5) 去除 26 个协变量, 包括来自白质、脑脊液的信号和 24 个 Friston 头动参数, 以减少生理和头动的污染. 由于前 人研究发现 Friston 24 参数模型 ${ }^{[42]}$ 在去除头动影响方 面比较有效 ${ }^{[43]}$, 故采用该模型来去除头动效应. 同 时, 也去除了线性趋势. (6) 空间标准化, 采用 SPM8 自带的 DARTEL (diffeomorphic anatomical registration through exponentiated Lie algebra $)^{[44]}$, 将扫描所 得到的结构图像标准化到 MNI (montreal neurological institute)标准空间. (7) 以 $4 \mathrm{~mm} \times 4 \mathrm{~mm} \times 4 \mathrm{~mm}$ 的全宽 半高(full width at half maximum, FWHM)进行高斯平 滑, 以增加信噪比.

为了减少头动可能带来的噪音, 将volume-level framewise displacement (FD) 作为被试头动参数 ${ }^{[45]}$. 将平均FD超过 2 个标准差以外的被试剔除 $(0.0686 \pm$ $0.0377, M \pm S D)^{[46]}$. 在168名被试中, 有7个被试由于 平均FD超过 0.144 而被删除.

(vii) ALFF 计算. 采用 DPARSFA 处理流程中调 用 REST (resting state fMRI data analysis toolkit, version 1.8)程序 ${ }^{[47]}$ 进行分析. 首先将全脑每一个体素的 时间序列进行傅里叶变换, 得到频域功率谱. 功率谱 的峰下面积可视为信号的能量. 对峰下面积开平方, 得到的值即可代表信号震荡的幅度. 同时, 将每个体 素在 $0.01 \sim 0.08 \mathrm{~Hz}$ 之间的频率平方根进行平均, 即为 ALFF 值 ${ }^{[14]}$. 为了标准化, 将每个体素的 ALFF 值减 去全脑的平均 ALFF 值并除以标准差, 最后得到每个 体素标准化的 ALFF 值.

(viii) 统计分析. 采用SPM8统计模块进行统计 分析. 首先进行多重回归分析. 以中国人幸福感量表 的得分为自变量, 以ALFF值为因变量, 采用全脑的 mask, 进行多重回归分析. 同时, 将性别、年龄和头 动平均 $\mathrm{FD}$ 值纳人协变量, 以剔除这些因素对结果的 影响. 多重回归分析结果通过AFNI软件中的AlphaSim 程序 (http://afni.nimh.nih.gov/pub/dist/doc/pro- 
gram_help/AlphaSim.html) 进行基于蒙特卡罗模拟 (Monte Carlo simulation)的多重比较校正. 结果得到一 个校正阈限为 $P<0.05$ 的结果图. 参数为: 单个体素 $P$ $=0.05$, 5000 simulations, FWHM $=4 \mathrm{~mm}$, cluster connection radius $r=5 \mathrm{~mm}$. 最后发现, 要达到统计显著 差异水平, 最小的cluster大小为 54 voxels $\left(1458 \mathrm{~mm}^{3}\right)$.

(ix) 行为与大脑的相关. 为了进一步了解脑区 的功能意义, 将与幸福感有显著相关的大脑区域的 平均 ALFF 值提取出来, 并与行为量表得分进行 Pearson 相关分析.

\section{2 结果}

\section{1 样本特征}

表 1 呈现的是本研究所选样本的描述性特征. 我 们还对各个量表进行了相关分析. 结果表明, 幸福感 量表得分与积极情感量表得分有显著正相关 $(r=0.60$, $P<0.001)$, 与消极情感量表得分有显著负相关 $(r=$ $-0.42, P<0.001)$, 并且与 RRS 量表的 2 个分量表均 有显著负相关(强迫性冥思: $r=-0.39, P<0.001$; 反
思, $r=-0.30, P<0.05)$. 该结果也验证了幸福感测量 的有效性.

\section{2 多重回归结果}

多重回归结果见图 1 和表 2 . 回归后发现, 左、 右侧的眶额皮层 (orbitofrontal cortex, OFC) 和左侧的 背外侧前额皮层 (dorsolateral prefrontal cortex, DLPFC), 以及右侧的尾状核(caudate)的 ALFF 值越 高, 幸福感水平越高。相反, 右侧的中扣带皮层 (middle cingulate gyrus, MCG), 左侧的顶上沟 (superior parietal lobule, SPL) 以及双侧的楔前叶的 ALFF 值越低, 幸福感水平越高.

\section{3 大脑与行为相关结果}

将与幸福感有显著相关脑区的平均 ALFF 值与 行为量表得分进行相关分析, 结果见表 3. 结果发现, 这些大脑区域和幸福感量表的得分都有显著相关 $(R \mathrm{~s}$ $>0.29, P \mathrm{~s}<0.001)$, 验证了我们脑成像上的发现. 此 外, 左、右侧的眶额皮层的 ALFF 值与积极情绪有显

表1 样本描述性特征 ${ }^{a)}$

Table 1 Demographics

\begin{tabular}{cccc}
\hline & $M$ & $S D$ & 区间 \\
\hline 年龄(年) & 20.90 & 1.64 & $18 \sim 26$ \\
头动 $(\mathrm{mm})$ & 0.061 & 0.026 & $0.023 \sim 0.142$ \\
中国人幸福感 $(n=159)$ & 2.41 & 0.38 & $1.52 \sim 3.48$ \\
积极情感 $(n=118)$ & 3.06 & 0.62 & $1.5 \sim 4.6$ \\
消极情感 $(n=118)$ & 1.90 & 0.50 & $1.0 \sim 3.3$ \\
RRS_强迫性冥思 $(n=72)$ & 2.06 & 0.49 & $1.0 \sim 3.6$ \\
RRS_沉思 $(n=72)$ & 2.19 & 0.55 & $1.2 \sim 4.0$ \\
\hline
\end{tabular}

a) RRS 为沉思反应量表(rumination response scales)

表 2 幸福感与 ALFF 的关系

Table 2 The relationships between Happiness and ALFF values

\begin{tabular}{|c|c|c|c|c|c|c|c|c|}
\hline \multirow{2}{*}{ 大脑区域 } & \multirow{2}{*}{ Side } & \multirow{2}{*}{ BAs } & \multicolumn{3}{|c|}{ MNI } & \multirow{2}{*}{$\begin{array}{c}\text { Voxel } \\
\text { size }\end{array}$} & \multirow{2}{*}{$\begin{array}{c}\text { Peak } \\
T \text {-value }{ }^{\text {a) }}\end{array}$} & \multirow{2}{*}{$P$ 值 } \\
\hline & & & $x$ & $y$ & $z$ & & & \\
\hline \multicolumn{9}{|l|}{ 正相关 } \\
\hline Left Orbitofrontal Cortex & $\mathrm{L}$ & 11 & -27 & 51 & -15 & 107 & 3.58 & $<10^{-3}$ \\
\hline Right Orbitofrontal Cortex & $\mathrm{R}$ & 11 & 18 & 42 & -18 & 55 & 4.22 & $<10^{-4}$ \\
\hline Dorsolateral prefrontal cortex & $\mathrm{L}$ & 9 & -48 & 27 & 39 & 68 & 3.78 & $<10^{-3}$ \\
\hline Caudate & $\mathrm{R}$ & & 15 & -6 & 18 & 97 & 3.89 & $<10^{-4}$ \\
\hline \multicolumn{9}{|l|}{ 负相关 } \\
\hline Middle Cingulate Gyrus & $\mathrm{R}$ & 31 & 6 & -42 & 42 & 82 & -3.55 & $<10^{-5}$ \\
\hline Superior Parietal Lobule & $\mathrm{L}$ & 7 & -33 & -66 & 60 & 194 & -4.14 & $<10^{-4}$ \\
\hline Precuneus & B & 7 & -3 & -60 & 33 & 89 & -4.51 & $<10^{-3}$ \\
\hline
\end{tabular}

a) $\mathrm{d} f=154$. Side 指的是大脑半球(B, 双侧; R, 右侧; L, 左侧). BAs 指的是 Brodmann 分区. 坐标指的是峰值点在 MNI (Montreal Neurological Institute)空间里的坐标 


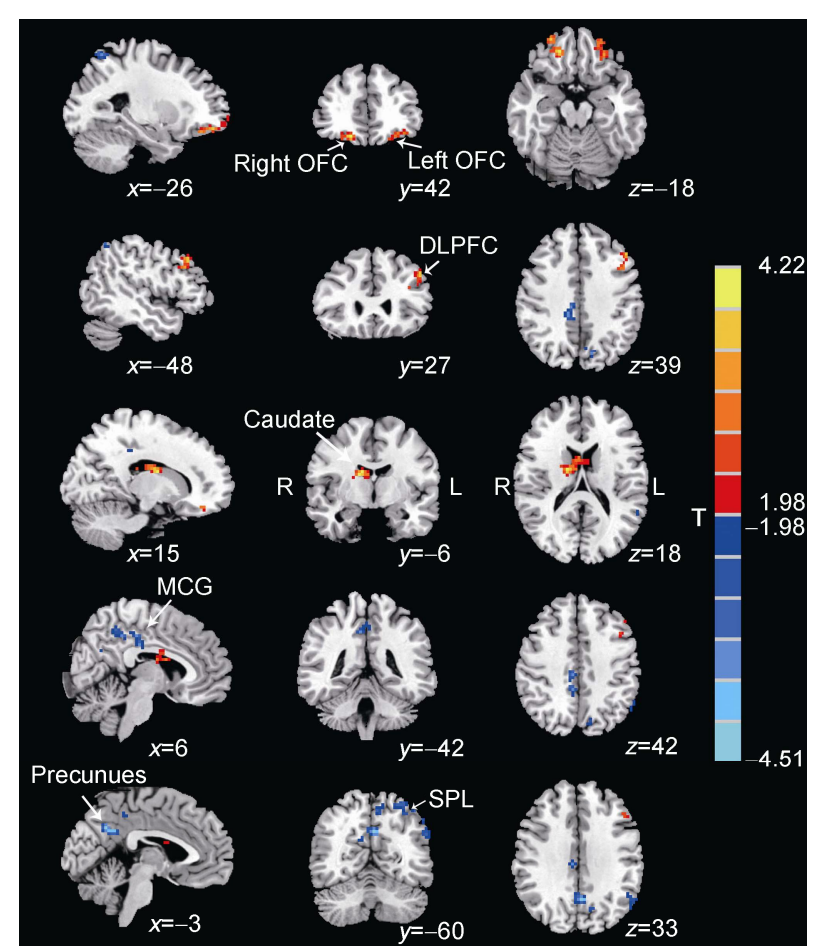

图 1 幸福感和全脑 ALFF 值的相关. 这是多重回归的结果 $(P<0.05$, AlphaSim corrected). 暖色调表示显著正相关, 冷色调表示显著负相 关. $\mathrm{R}$ 表示的是右半球, 而 $\mathrm{L}$ 表示的是左半球.

Figure 1 Regions showing significant correlations between ALFF values of the whole brain and happiness (multiple regressions, $P<0.05$, AlphaSim corrected). Warm colors indicate the positive correlations and cold colors indicate the negative correlations $(\mathrm{R}=$ right and $\mathrm{L}=$ left $)$. Abbreviations: Left OFC, left orbitofrontal cortex; Right OFC, right orbitofrontal cortex; DLPFC, dorsolateral prefrontal cortex; MCG, middle cingulate gyrus; spl, superior parietal lobule

著正相关 $(R \mathrm{~s}>0.30, P \mathrm{~s}<0.001)$, 背外侧前额皮层与 负性情绪有显著负相关 $(r=-0.21, P<0.05)$, 以及右 侧的尾状核与积极情绪有边缘正相关 $(r=-0.17, P=$ 0.07). 中扣带皮层, 顶上沟以及楔前叶的 ALFF 值也 都与积极情绪呈显著负相关 $(R \mathrm{~s}>-0.24, P \mathrm{~s}<0.01)$. 并且, 除了楔前叶与反思分量表不显著之外, 这些脑 区均与 RRS 的两个分量表有显著或边缘正相关.

\section{3 讨论}

本研究首次采用静息态功能磁共振中的 ALFF 技术来研究幸福感与大脑自发神经活动的关系. 结 果发现, 大脑静息态功能磁共振的低频振幅与幸福 感有着密切的关系. 具体地说, 左、右侧眀额皮层和 左侧背外侧前额皮层, 以及右侧尾状核的 ALFF 值越 高, 幸福感水平越高. 并且, 这些区域的平均 ALFF
值都与正性情绪或负性情绪有显著相关. 相反的是, 相对位于后部的右侧中扣带皮层, 左侧顶上沟以及 双侧楔前叶的 ALFF 值越低, 幸福感水平越高. 这些 后部区域的 ALFF 值都与积极情绪有显著负相关. 并 且, 除了楔前叶与反思没有显著相关之外, 其他脑区 均与倾向量表的 2 个分量表有显著正相关. ALFF 从 能量的角度反映了大脑各区域在静息状态下自发神 经活动水平的高低 ${ }^{[14]}$, 本研究发现的 ALFF与幸福感 之间的关系可能有助于理解高低幸福感个体之间在 认知和动机上存在的系统差异.

本研究发现, 尾状核的 ALFF 值随着幸福感的增 加而升高, 并且与积极情绪有边缘显著正相关. 尾状 核是纹状体(属于基底核)中的一部分. 鉴于 ALFF 反 映了自发神经活动水平的特性, 可以认为, 低幸福感 个体在静息状态下纹状体的自发神经活动能量较低. 纹状体是参与奖赏或快乐的体验和期待的核心结 构 $^{[25]}$. 例如, 当被试在即时体验快乐 ${ }^{[48]}$, 或在期待将 来快乐事件 ${ }^{[49]}$ 时, 纹状体的激活水平增加. 能够体 验和期待快乐感是个体的一种重要的适应能力. 对 快乐的加工能够引发积极情绪, 促进目标行为的达 成 ${ }^{[50]}$. 反之, 体验不到或不期待快乐, 则大多会造成 不幸福, 如抑郁、精神分裂和各种成瘾 ${ }^{[24]}$. 该结果也 和我们之前发现高低幸福感个体在纹状体的局部一 致性上存在差异的结果一致 ${ }^{[26]}$. 总的来说, 这样的 结果可能表明了高幸福感个体在体验和期待快乐的 能力方面要优于低幸福感个体.

此外, 本研究还发现了左右侧的眀额皮层和背 外侧前额皮层的 ALFF 值也随着幸福感的增加而升 高, 并且它们都与正性情绪或负性情绪显著正相关. 睈额皮层, 是大脑参与情绪加工神经网络的重要组 成部分, 主要参与了由奖赏所带来的愉悦体验, 如对 美味的食物所带来的主观愉悦价值进行编码 ${ }^{[51]}$. 以 往研究发现, 眀额皮层的灰质体积与外向型人格特 征成正比 ${ }^{[52]}$, 而该皮层灰质体积的减少, 还与心理 障碍有关, 如抑郁症等 ${ }^{[53]}$. 重要的是, 眀额皮层, 特 别是外侧的眶额皮层和背外侧前额皮层都与认知控 制及情绪调节有关 ${ }^{[54]}$. 这些区域参与了对不合适的 信息、情绪和行为的抑制, 从而最终促使个体达成目 标行为. 例如, 基于任务的 fMRI 研究发现, 当被试 试图去抑制由悲伤电影引起的伤心情绪时, 眭额皮 层和背外侧前额皮层都会被激活 ${ }^{[5]}$. 并且, 有研究 
表 3 有显著差异脑区与行为量表之间的相关 ${ }^{a)}$

Table 3 The correlations between the behavioral scales and the ALFF values of regions showing significant effect

\begin{tabular}{cccccrr}
\hline & & 幸福感 $(n=159)$ & 积极情绪 $(n=118)$ & 消极情绪 $(n=118)$ & RRS_强迫性冥思 $(n=72)$ & RSS_反思 $(n=72)$ \\
\hline \multirow{2}{*}{ Left OFC } & $r$ & $0.338^{* *}$ & $0.369^{* * *}$ & -0.006 & 0.002 & -0.041 \\
& $P$ & 0.000 & 0.000 & 0.948 & 0.989 & 0.735 \\
Right OFC & $r$ & $0.294^{* *}$ & $0.310^{* *}$ & -0.093 & -0.093 & -0.043 \\
& $P$ & 0.000 & 0.001 & 0.316 & 0.437 & 0.720 \\
DLPFC & $r$ & $0.376^{* *}$ & 0.128 & $-0.215^{*}$ & 0.011 & -0.050 \\
& $P$ & 0.000 & 0.167 & 0.020 & 0.927 & 0.677 \\
Caudate & $r$ & $0.350^{* *}$ & 0.167 & -0.137 & -0.059 & 0.018 \\
\multirow{2}{*}{ MCG } & $P$ & 0.000 & 0.070 & 0.138 & 0.621 & 0.881 \\
& $r$ & $-0.359^{* *}$ & $-0.240^{* *}$ & 0.146 & $0.394^{* *}$ & $0.333^{* *}$ \\
& $P$ & 0.000 & 0.009 & 0.114 & 0.001 & 0.004 \\
\multirow{2}{*}{ SPL } & $r$ & $-0.501^{* *}$ & $-0.273^{* *}$ & 0.118 & $0.291^{*}$ & 0.212 \\
& $P$ & 0.000 & 0.003 & 0.202 & 0.013 & 0.074 \\
& $r$ & $-0.447^{* *}$ & $-0.235^{*}$ & 0.016 & $0.263^{*}$ & 0.147 \\
\hline
\end{tabular}

a) $*, P<0.05 ; * *, P<0.01$. RRS, rumination response scales; Left OFC, left orbitofrontal cortex; Right OFC, right orbitofrontal cortex; DLPFC, dorsolateral prefrontal cortex; MCG, middle cingulate gyrus; SPL, superior parietal lobule

还发现被试使用再评策略去减少而不是增加负性情 绪时, 外侧眶额皮层激活程度更大; 相反, 如果被试 使用再评策略去增加负性情绪时, 背外侧前额皮层 激活程度更大 ${ }^{[56]}$. 这有可能说明背外侧前额皮层和 眶额皮层在情绪调节上的作用是不一样的，背外侧 前额皮层更多的是保持或者增强当前的情绪体验, 而眀额皮层更多参与抑制或降低负性情绪 ${ }^{[54]}$. 因此, 高幸福感个体在眀额皮层和背外侧前额皮层的 ALFF 值升高有可能说明他们有着完好的情绪调节能力. 这也与以往的幸福行为研究是一致的, 如 $\mathrm{Pe}$ 等人 ${ }^{[23]}$ 发现高幸福感个体能够有效地调节积极信息, Diener 和 Seligman ${ }^{[2]}$ 的研究也发现高幸福感个体有着较高 的积极情绪.

本研究的另一个发现是双侧楔前叶的 ALFF 值 随着幸福感的升高而降低, 并且其平均 ALFF 值与强 迫性冥思有显著正相关. 楔前叶是默认网络的一个 重要结构. 楔前叶一般被认为参与了与自我有关的 情节记忆和自我反思 ${ }^{[57]}$. 以往对抑郁症的静息态功 能连接研究发现, 抑郁症患者的楔前叶的功能连接 要高于健康被试 ${ }^{[58]}$. 因此, 低幸福感个体在这些区 域有着相对较高的 ALFF 值, 可能表明了这些个体过 多地进行了与自我有关的思考, 很难从自我思考中 抽离出来. 这种推测也与以往对幸福的研究是一致 的 ${ }^{[30,31]}$. 例如, 低幸福个体沉浸在自己失败的体验中, 很难集中注意力于当前任务, 从而影响了作业成 绩 [31].
尽管本研究得到了一些有趣的结果, 但也存在 一定的局限性. 首先, 本研究只是一个横向的相关研 究, 不能明确地给出大脑与幸福感之间的因果关系, 以后可以采用更为精巧的实验设计来研究它们之间 的因果关系. 其次, 尽管本研究所测量到的幸福感是 一种特质(trait), 但是, 由于幸福感的复杂性, 静息 态 ALFF 与幸福感的关系难免会受到被试状态的影 响. 如何分别研究被试的特质幸福和状态幸福与大 脑的关系, 这也是将来值得研究的问题. 最后, 本研 究只采用 ALFF 这个静息态指标, 研究的只是单一磁 共振信号的特点, 以后应该研究多个磁共振信号在 时间序列上的关系, 如采用功能连接和有效连接等 指标来研究各个脑区、网络之间的连接与幸福感的关 系 ${ }^{[59]}$ ，以及 VMHC (voxel-mirrored homotopic correlation ${ }^{[60]}$ 指标来研究大脑左右半球之间的功能连接 与幸福感之间的关系, 以更为全面地揭示幸福感与 大脑的关系.

\section{4 结论}

幸福感与大脑的关系是一个有着重要意义的问 题. 本研究采用静息态功能磁共振中的低频振幅指 标来研究幸福感与大脑自发神经活动之间的关系. 眶额皮层和背外侧前额皮层, 以及尾状核的 ALFF值 随着幸福感的增加而提高. 并且, 这些区域的平均 ALFF值都与正性情绪或负性情绪有显著相关. 相反, 楔前叶，中扣带皮层以及顶上沟的 ALFF 值随着幸福 
感的提高而降低. 这些区域的 ALFF 值不仅与积极情 绪有显著负相关, 还与沉思倾向有正相关. 这些结果 可能反映了高低幸福感个体在自我加工、快乐加工和
情绪加工等方面存在的差异. 本研究的发现有助于 理解幸福感与大脑的关系，也能够为以后提高幸福 感的实践提供理论上的指导.

\section{参考文献}

1 Diener E. Subjective well-being: The science of happiness and a proposal for a national index. Am Psychol, 2000, 55: 34-43

2 Diener E, Seligman M E P. Very happy people. Psychol Sci, 2002, 13: 81-84

3 Diener E. The remarkable changes in the science of subjective well-being. Perspect Psychol Sci, 2013, 8: 663-666

4 Lyubomirsky S. Why are some people happier than others? The role of cognitive and motivational processes in well-being. Am Psychol, 2001, 56: 239-249

5 Kringelbach M L, Berridge K C. Towards a functional neuroanatomy of pleasure and happiness. Trends Cogn Sci, 2009, 13: 479-487

6 Cunningham W A, Kirkland T. The joyful, yet balanced, amygdala: Moderated responses to positive but not negative stimuli in trait happiness. Soc Cogn Affect Neurosci, 2014, 9: 760-766

7 Heller A S, van Reekum C M, Schaefer S M, et al. Sustained striatal activity predicts eudaimonic well-being and cortisol output. Psychol Sci,, 2013, 24: 2191-2200

8 Biswal B, Yetkin F Z, Haughton V M, et al. Functional connectivity in the motor cortex of resting human brain using echo-planar mri. Magnet Reson Med, 1995, 34: 537-541

9 Shehzad Z, Kelly A M, Reiss P T, et al. The resting brain: Unconstrained yet reliable. Cereb Cortex, 2009, 19: 2209-2229

10 Zuo X N, Di Martino A, Kelly C, et al. The oscillating brain: Complex and reliable. NeuroImage, 2010, 49: 1432-1445

11 Broyd S J, Demanuele C, Debener S, et al. Default-mode brain dysfunction in mental disorders: A systematic review. Neurosci Biobehavr, 2009, 33: 279-296

12 Whitfield-Gabrieli S, Ford J M. Default mode network activity and connectivity in psychopathology. Ann Rev Clin Psych, 2012, 8: 49-76

13 Zuo X N, Zhang Z, He Y, et al. The human functional connectome: Its methodology, developmental trajectory and behavioral association (in Chinese). Chin Sci Bull (Chin Ver), 2012, 57: 3399-3413 [左西年, 张喆, 贺永, 等. 人脑功能连接组: 方法学、发展轨线和行为关 联. 科学通报, 2012, 57: 3399-3413]

14 Zang Y F, He Y, Zhu C Z, et al. Altered baseline brain activity in children with adhd revealed by resting-state functional mri. Brain Dev, 2007, 29: 83-91

15 Yang H, Long X Y, Yang Y, et al. Amplitude of low frequency fluctuation within visual areas revealed by resting-state functional mri. NeuroImage, 2007, 36: 144-152

16 Wei L, Duan X, Zheng C, et al. Specific frequency bands of amplitude low-frequency oscillation encodes personality. Hum Brain Mapp, 2014, 35: 331-339

17 Liu H, Liao J, Jiang W, et al. Changes in low-frequency fluctuations in patients with antisocial personality disorder revealed by resting-state functional mri. PLoS ONE, 2014, 9: e89790

18 He Y, Wang L, Zang Y, et al. Regional coherence changes in the early stages of alzheimer's disease: A combined structural and resting-state functional mri study. NeuroImage, 2007, 35: 488-500

19 Han Y, Wang J, Zhao Z, et al. Frequency-dependent changes in the amplitude of low-frequency fluctuations in amnestic mild cognitive impairment: A resting-state fmri study. NeuroImage, 2011, 55: 287-295

20 Hoptman M J, Zuo X N, Butler P D, et al. Amplitude of low-frequency oscillations in schizophrenia: A resting state fmri study. Schizophr Res, 2010, 117: 13-20

21 Yu R, Chien Y L, Wang H L, et al. Frequency-specific alternations in the amplitude of low-frequency fluctuations in schizophrenia. Hum Brain Mapp, 2014, 35: 627-637

22 Guo W B, Liu F, Xue Z M, et al. Alterations of the amplitude of low-frequency fluctuations in treatment-resistant and treatment-response depression: A resting-state fmri study. Prog Neuropsychopharmacol Biol Psychiatry, 2012, 37: 153-160

23 Pe M L, Koval P, Kuppens P. Executive well-being: Updating of positive stimuli in working memory is associated with subjective well-being. Cognition, 2013, 126: 335-340

24 Berridge K C, Kringelbach M L. Affective neuroscience of pleasure: Reward in humans and animals. Psychopharmacology, 2008, 199: $457-480$ 
26 Luo Y, Huang X, Yang Z, et al. Regional homogeneity of intrinsic brain activity in happy and unhappy individuals. PLoS ONE, 2014, 9: e85181

27 Ochsner K N, Gross J J. The cognitive control of emotion. Trends Cogn Sci, 2005, 9: 242-249

28 Tang G Q, Huang M E. Diverse consequences of negative emotional responses between high and low happiness people (in Chinese). Acta Psychol Sin, 2012, 44:1086-1099 [唐涂琦，黄敏儿. 高低幸福感人群的负情绪特点：生理和表情的依据. 心理学报，2012，44: 1086-1099]

29 Nolen-Hoeksema S, Wisco B E, Lyubomirsky S. Rethinking rumination. Perspect Psychol Sci, 2008, 3: 400-424

30 Killingsworth M A, Gilbert D T. A wandering mind is an unhappy mind. Science, 2010, 330: 932-932

31 Lyubomirsky S, Boehm J K, Kasri F, et al. The cognitive and hedonic costs of dwelling on achievement-related negative experiences: Implications for enduring happiness and unhappiness. Emotion, 2011, 11: 1152-1167

32 Mason M F, Norton M I, Van Horn J D, et al. Wandering minds: The default network and stimulus-independent thought. Science, 2007, 315: 393-395

33 Weissman D H, Roberts K C, Visscher K M, et al. The neural bases of momentary lapses in attention. Nat Neurosci, 2006, 9: 971-978

34 Buckner R L, Andrews-Hanna J R, Schacter D L. The brain's default network - anatomy, function, and relevance to disease. Year in Cognitive Neuroscience, 2008, 2008, 1124: 1-38

35 Raichle M E, MacLeod A M, Snyder A Z, et al. A default mode of brain function. Proc Natl Acad Sci USA, 2001, 98: 676-682

36 Lu L, Shih J B, Lin Y Y, et al. Personal and environmental correlates of happiness. Personal Individ Diff, 1997, 23: 453-462

37 Watson D, Clark L A, Tellegen A. Development and validation of brief measures of positive and negative affect - the panas scales. J Pers Soc Psychol, 1988, 54: 1063-1070

38 Huang L, Yang T, Ji Z. Applicability of the positive and negative affect scale in Chinese (in Chinese). Chin Mental Health J, 2003, 17: 54-56 [黄丽，杨廷忠，季忠民. 正性负性情绪量表的中国人群适用性研究. 中国心理卫生杂志, 2003, 17: 54-56]

39 Treynor W, Gonzalez R, Nolen-Hoeksema S. Rumination reconsidered: A psychometric analysis. Cog Therapy Res, 2003, 27: 247-259

40 Yang J, Ling Y, Xiao J, et al. The Chinese version of ruminative responses scale in high school students: Its reliability and validity (in Chinese). Chin J Clin Psychol, 2009, 27-28: 31 [杨娟，凌宇，肖晶，等. 沉思反应量表中文版在高中生中初步运用结果分析. 中国临 床心理学杂志, 2009, 27-28: 31]

41 Chao-Gan Y, Yu-Feng Z. Dparsf: A matlab toolbox for "pipeline” data analysis of resting-state fmri. Front Syst Neurosci, 2010, 4: 13

42 Friston K J, Williams S, Howard R, et al. Movement-related effects in fmri time-series. Magnet Reson Med, 1996, 35: 346-355

43 Yan C G, Cheung B, Kelly C, et al. A comprehensive assessment of regional variation in the impact of head micromovements on functional connectomics. NeuroImage, 2013, 76: 183-201

44 Ashburner J. A fast diffeomorphic image registration algorithm. NeuroImage, 2007, 38: 95-113

45 Jenkinson M, Bannister P, Brady M, et al. Improved optimization for the robust and accurate linear registration and motion correction of brain images. NeuroImage, 2002, 17: 825-841

46 Yan C G, Craddock R C, Zuo X N, et al. Standardizing the intrinsic brain: Towards robust measurement of inter-individual variation in 1000 functional connectomes. NeuroImage, 2013, 80: 246-262

47 Song X W, Dong Z Y, Long X Y, et al. Rest: A toolkit for resting-state functional magnetic resonance imaging data processing. PLoS One, 2011, 6: e25031

48 Tanaka SC, Doya K, Okada G, et al. Prediction of immediate and future rewards differentially recruits cortico-basal ganglia loops. Nat Neurosci, 2004, 7: 887-893

49 Knutson B, Adams C M, Fong G W, et al. Anticipation of increasing monetary reward selectively recruits nucleus accumbens. J Neurosci, 2001, 21: RC159

50 Schultz W. Neural coding of basic reward terms of animal learning theory, game theory, microeconomics and behavioural ecology. Curr Opin Neurobiol, 2004, 14: 139-147

51 Kringelbach M L. The human orbitofrontal cortex: Linking reward to hedonic experience. Nat Rev Neurosci, 2005, 6: 691-702

52 DeYoung C G, Hirsh J B, Shane M S, et al. Testing predictions from personality neuroscience. Brain structure and the big five. Psychol Sci, 2010, 21: 820-828

53 Bremner J D, Vythilingam M, Vermetten E, et al. Reduced volume of orbitofrontal cortex in major depression. Biol Psychiatry, 2002, 51: 273-279

54 Hooker C I, Knight R T. The role of lateral orbitofrontal cortex in the inhibitory control of emotion. In: Zald D, Rauch S, eds. The Orbitofrontal Cortex. New York: Oxford University Press, 2006. 307-323

55 Lévesque J, Eugène F, Joanette Y, et al. Neural circuitry underlying voluntary suppression of sadness. Biol Psychiat, 2003, 53: 502-510 
56 Ochsner K N, Ray R D, Cooper J C, et al. For better or for worse: Neural systems supporting the cognitive down- and up-regulation of negative emotion. NeuroImage, 2004, 23: 483-499

57 Fransson P, Marrelec G. The precuneus/posterior cingulate cortex plays a pivotal role in the default mode network: Evidence from a partial correlation network analysis. NeuroImage, 2008, 42: 1178-1184

58 Greicius M D, Flores B H, Menon V, et al. Resting-state functional connectivity in major depression: Abnormally increased contributions from subgenual cingulate cortex and thalamus. Biol Psychiat, 2007, 62: 429-437

59 Buckner R L, Krienen F M, Yeo B T T. Opportunities and limitations of intrinsic functional connectivity mri. Nat Neurosci, 2013, 16: $832-837$

60 Zuo X N, Kelly C, Di Martino A, et al. Growing together and growing apart: Regional and sex differences in the lifespan developmental trajectories of functional homotopy. J Neurosci, 2010, 30: 15034-15043

\title{
Amplitude of low-frequency fluctuations in happiness: A resting-state fMRI study
}

\author{
LUO YangMei ${ }^{1,2}$, LI BaoLin $^{1}$, LIU Jie ${ }^{1}$, BI ChongZeng ${ }^{1} \&$ HUANG XiTing ${ }^{1}$ \\ ${ }^{1}$ Faculty of Psychology, Key Laboratory of Cognition and Personality, Ministry of Education, Southwest University, Chongqing 400715, China; \\ ${ }^{2}$ School of Psychology, Shaanxi Normal University, Xi'an 710062, China
}

Happiness is a universal pursuit; however, not everyone is equipped with the same ability to be happy. Therefore, it is important to investigate the individual differences that affect happiness. Little attention has been devoted to understanding the neural basis of individual differences in happiness. We thus used the amplitude of low-frequency fluctuations (ALFF) in resting-state functional magnetic resonance imaging (fMRI) to study the relationship between happiness and spontaneous brain activity in 159 healthy participants. The results showed that ALFF values for the frontal, parietal and parts of subcortical areas were associated with happiness. Specifically, the ALFF values in the left and right orbitofrontal cortex, left dorsolateral prefrontal cortex, and right caudate positively predicted happiness. Furthermore, these areas were significantly correlated with participants' emotional states. By contrast, the ALFF values in the right middle cingulate gyrus, superior parietal lobule, and bilateral precuneus negatively predicted happiness, and were not only correlated with emotions, but also with rumination. These findings indicate that there may be underlying systematic differences in self-reflection, reward processing, emotion, and emotional regulation between happy and unhappy individuals.

happiness, resting-state fMRI, ALFF, pleasure, emotional regulation, rumination

doi: 10.1360/N972014-00700 